\title{
Competemcias, características y ambiente institucional en educadores de albergues infantiles
}

Competences, characteristics and institutional environment of educators at children shelters

\author{
Martha E. Serrano Arias \\ Martha Frías Armenta \\ Universidad de Sonora
}

\begin{abstract}
Resumen
En México, los albergues infantiles son requeridos para garantizar niveles mínimos de bienestar (Gobierno de México, 2002) a niños víctimas de violencia y abandono. En estos albergues, la presencia del educador que cuida de ellos es de suma importancia y ha de ser capacitado y supervisado (Gobierno de Brasil, 2007). A este respecto, la educación social sustenta en su teoría el perfil de un educador capaz de atender de manera directa a los grupos vulnerables, incluidos niños y adolescentes (AIEJI, 2007). De aquí se desprende que el objetivo del presente estudio sea el análisis de las competencias, características personales y ambiente institucional de los educadores que laboran en albergues de Hermosillo, Sonora, México. Los participantes fueron 117 educadores de 10 instituciones de asistencia social, dedicadas al albergue de menores maltratados o en situación de riesgo. El instrumento fue una escala de evaluación basada en los indicadores del Libro Blanco (ANECA, 2005). Los participantes fueron entrevistados en los albergues donde se encontraban trabajando. Se computaron estadísticas univariadas alfas de Cronbach y coeficientes de correlación para el análisis de datos. Las correlaciones significativas fueron encontradas entre lenguaje, razonamiento y funciones educativas de los educadores (.26) y con compromiso con la propia identidad (26); la satisfacción de necesidades se relacionó con compromiso con la propia identidad (.22) con lenguaje y razonamiento (.39); y último grado de estudios con habilidades interpersonales (26). La edad se correlacionó con la antigüedad (.48), el último grado de estudios (-.19), satisfacción de necesidades (.25) y lenguaje y razonamiento (.19). Estos resultados muestran la importancia del ambiente institucional en el desarrollo de las funciones educativas y el compromiso con la institución de los educadores.
\end{abstract}

Palabras Clave: Competencias, educadores sociales, albergues, menores

Nota del autor:

Martha E. Serrano Arias, Doctorado en Ciencias Sociales, Universidad de Sonora; Martha Frías Armenta, División de Ciencias Sociales, Universidad de Sonora.

La correspondencia en relación con este artículo debe dirigirse a Martha E. Serrano Arias, Doctorado en Ciencias Sociales, Universidad de Sonora, Blvd. Luis Encinas y Rosales, s/n, Col. Centro, C.P. 83000, Hermosillo, Sonora, México.

Correo electrónico: me-serrano@ hotmail.com 


\section{Abstract}

In Mexico, shelters for abused or neglected children are required to guarantee minimum levels of wellbeing (Gobierno de México, 2002). The work that educators engage in at these shelters is very important and has to be qualified and supervised (Gobierno de Brasil, 2007). Regarding this matter, the theory of Social Education stresses the relevance of an educator as someone who is able to taking care of vulnerable groups like children and teenagers (AIEJI, 2007). Therefore, the objective of this study was to analyze the educators' competency, their personal characteristics, and institutional environment at public and private shelters in Hermosillo, Sonora, Mexico. One hundred-and-seventeen educators at seven institutions devoted to shelter abused or poor children participated in the study. The instrument was an evaluation scale based on the established indicators of the White Book for a degree in Pedagogy and Social Education (Libro Blanco, ANECA, 2005). The participants were interviewed in the shelters they worked at. Univariate statistics, Cronbach's alphas and correlations were obtained to analyze the data. Language \& reasoning resulted significantly correlated with educative functions (.26) and with commitment with own identity (26); satisfaction of needs and commitment also resulted significantly related to own identity (.22) and to language and reasoning (.39). One more significant correlation resulted between schooling and interpersonal abilities (26). Age was correlated with time on the job (.48), schooling (-.19), satisfaction of needs (.25) and language and reasoning (.19). These results show the importance of institutional environments in the development of educational functions and institutional commitment of educators.

Keywords: Competencies, social educators, shelters, children

Los padres desempeñan tareas críticas en el desarrollo de los niños, ya que les ayudan a adaptarse al ambiente social y físico (Bradley, 2002). Sin embargo, alrededor del mundo existen millones de niños privados de uno o ambos padres (UNICEF, 2007). Más de dos millones de éstos se encuentran "bajo tutela institucional" (UNICEF, 2009, p.19) a veces por períodos innecesariamente largos. Estos niños tienen más probabilidades de sufrir discriminación, cuidados inadecuados y malos tratos. El Fondo Internacional de las Naciones Unidas de Auxilio a la Infancia (UNICEF, 2009), estima que en América Latina y el Caribe hay aproximadamente 200, 000 niños al cuidado de las instituciones. Sin embargo, esta institución reporta también que la cifra es muy inferior a la realidad, ya que aún no se cuenta con estimaciones exactas de cada país.

A raíz de los problemas legales en que han incurrido algunas instituciones mexicanas de asistencia social como la Iglesia Cristiana Restaurada, las asociaciones civiles de Reintegración Social, Alas y los albergues Casitas del Sur (Contreras, 2009) se propone una
Norma Oficial Mexicana (NOM) para determinar las condiciones de operación de los albergues infantiles. Ésta, "incluiría no sólo aspectos de nutrición, educación y atención médica para los infantes, sino también aquellos aspectos relacionados con capacitación del personal que los atenderá" (Cámara de Diputados, 2009, p.15). En el estado de Sonora, algunas instituciones que proveen de albergue a los menores, se encuentran bajo la supervisión de la Junta de Asistencia Privada del Estado de Sonora (JAPS) y otras bajo la supervisión directa de la Ley de Salud para el Estado (1992). No obstante, no se ha encontrado una base de datos estatal que las contenga a todas, el personal con que trabajan o la capacitación con que cuentan.

El 20 de noviembre de 2009, la UNICEF aprobó un anteproyecto de directrices para el cuidado de los menores en albergues, el cual estipula los contenidos mínimos en las legislaciones nacionales. Estos criterios estándar servirán para la selección, monitoreo y supervisión de los educadores, así como para proveer de un adecuado reconocimiento de estos profesionales. Así mismo, hace énfasis en 
la necesidad de que los educadores entiendan la importancia de su propio rol en el desarrollo de una relación política, segura y de abrigo con el menor (Gobierno de Brasil, 2007). Sin embargo, es difícil llevar a cabo esta labor cuando, en el ámbito nacional, no se les conoce ni reconoce.

En el estado de Sonora se han realizado estudios e investigaciones sobre el rol que diferentes profesionales desempeñan en los albergues (Cervantes, 1997; Chávez, 1986); esto a partir de un punto de vista psicológico y de trabajo social. Desde la perspectiva educativa no se han realizado investigaciones sobre las características del educador que atiende de manera directa a los menores. Las funciones de estos sujetos sociales y el impacto que sus acciones puedan tener sobre los niños constituyen temas poco explorados. Se considera necesario disponer de un marco conceptual operativo para la intervención en dichos albergues que defina las fases, responsabilidades y tareas de los involucrados en este proceso (Cruz, 2009). Esta investigación evalúa bajo el esquema teórico de educación social, la formación de las personas que atienden a los niños de los albergues. Desde el punto de vista metodológico operacionaliza las competencias generales de los educadores sociales y ofrece una medición conceptual de éstas.

\section{Influencia del ambiente institucional}

Los albergues infantiles son lugares imprescindibles dentro del sistema de protección a la infancia (Fuertes \& Fernández del Valle, 2001) dedicados a la atención de una población a la que se le ha negado un clima familiar que facilite su desarrollo (Comunidad Valenciana, 1991). De acuerdo con Schreiner (2009), existen algunos estudios sobre las consecuencias que produce la institucionalización prolongada y desde temprana edad en la vida adulta de los niños en albergues. Éstos muestran escasas capacidades para realizar elecciones saludables para sí mismos y un impacto en su vida en el área productiva, familiar y social. Es importante por eso poner énfasis en la calidad de la relación que surge entre el niño o adolescente y el ambiente en el que se desarrolla, del cual el educador que lo atiende forma parte (Fernández del Valle, 1992). En este sentido, el ambiente establece lo que los niños pueden ver, oír, y hacer (Bradley, 2002). Igualmente, puede estimular o desalentar el desarrollo de los niños. Además, la inestabilidad ambiental puede llevar a conductas desadaptadas en los menores (Wachs, 2000). Un estudio realizado en Yucatán encontró que el rendimiento escolar de los niños que provenían de albergues era menor al de los niños que vivían con sus propios padres (Reyes Mendoza \& Solis Lugo, 1999). Retrasos en el desarrollo, depresión, ansiedad y dificultades en el aprendizaje fueron comunes en niños que vivían en estas instituciones (Bassuk \& Rubin, 1987). Los menores de los albergues, comparados con un grupo control, sufrieron más retraso en el desarrollo y más problemas emocionales y cognitivos (Rescorla, Parker, \& Stolley, 1991). Además, desde la perspectiva de los cuidadores de los albergues y el personal de la escuela, los niños de albergues presentan barreras para educarse como la carencia de apoyo parental, ropa y transporte inapropiado (Dupper \& Halter, 1994). Bassuk, \& Rosenberg (1989) compararon a niños pobres que vivían con sus madres con niños de albergues y encontraron que los dos grupos presentaban retraso en el desarrollo en edad preescolar; sin embargo, los que se encontraban institucionalizados exhibían un mayor retardo. Igualmente, los niños de albergue, medidos con la Lista de Chequeo Conductual de Achenbach (1992), manifestaban más problemas de internalización y externalización (Bassuk, Weinreb, Dawson, Perloff, \& Buckner, 1997).

La adolescencia y juventud, más que otras etapas, experimentan con mayor intensidad el medio físico y social en el cual se encuentran insertos (Melendro, 2007). Es por esto que el albergue ha de ofrecerles un ambiente sano, abierto y sin prejuicios en el que la persona pueda expresarse tal y como es sin temor a sentirse rechazada (Mondragón \& Trigueros, 2004). En la medida que el ambiente físico y social permite y estimula su participación, tanto conjuntamente con un adulto como por sí mismo, el potencial evolutivo del entorno aumenta. Por el contrario, las condiciones laborales inadecuadas pueden tener una influencia negativa en el desarrollo de 
la práctica educativa y en la situación profesional (Cruz, 2009). Los menores se tornan entonces vulnerables debido a la carencia de recursos personales y a la "ausencia o la presencia limitada de un entorno social acogedor, vincular, que ofrezca los apoyos necesarios en caso de crisis o conflictos" (Melendro, 2007, p. 34).

La importancia de la evaluación del ambiente se centra en la valoración de los efectos de la pluralidad de variables de las instituciones. Para Fernández del Valle (1992) estas variables están constituidas por: a) las funciones arquitectónicas; b) los datos sociodemográficos de la población; c) las funciones organizativas que incluyen el funcionamiento de la dependencia administrativa, del personal, y la suficiencia de plantillas; d) funciones psicosociales (aspectos del ambiente estudiados a través de la percepción de los habitantes); e) funciones conductuales; y f) interacciones entre los habitantes y con las personas del exterior. Otros autores (Redondo, Muñoz, \& Torres Gómez, 1998) hacen énfasis de manera particular en la interacción establecida entre el personal educativo y el menor. En la valoración realizada por los exresidentes hacia los centros (García-Barriocanal, De la Herrán, \& Imaña, 2007) se estima como positivo el apoyo y buen trato recibido por parte de los educadores, la educación recibida, las semejanzas entre el albergue y un ambiente familiar, y el ambiente estructurado con normas claras y espacios personalizados.

Para que los jóvenes se incorporen a una dinámica de proximidad y afecto que les ayude a establecer esta relación duradera con sus educadores es necesario mantener un clima de respeto (Melendro, 2007), cálido y acogedor (Bravo \& Fernández del Valle, 2009; Nesmith, 2006) que facilite "situaciones que permitan y estimulen las relaciones interpersonales y contribuyan a que la infancia acogida construya una imagen fiel y positiva de sí misma" (Cruz, 2009, p. 589). El niño o adolescente ha de sentir el valor de la estabilidad y consistencia en los cuidados del educador (Papalia, Wendkos, \& Duskin, 2001) y recibir reacciones sanas y espontáneas hacia su conducta, de tal manera que pueda realizar los ajustes personales y de comportamiento necesarios para alcanzar una convivencia gratificante que le permita generar, a partir de aquí, "esquemas relacionales nuevos y utilizables" para el futuro (Mondragón \& Trigueros, 2004, p. 196).

\section{Tendencia educativa y buenas prácticas}

Con el paso del tiempo, se ha visto la necesidad de cambiar la perspectiva del trabajo de los albergues, de un modelo basado en la asistencia social centrado únicamente en las carencias, a uno educativo (Fernández del Valle \& Fuertes, 2007) basado en el bienestar del niño (Cruz, 2009). Entre las prácticas identificadas por algunos autores (Arruabarrena, 2001; Bravo \& Fernández del Valle, 2009; Melendro, 2010; Mondragón \& Trigueros, 2004) como buenas y necesarias para el funcionamiento del albergue, se encuentran: la estabilidad de los equipos educativos, el trabajo con grupos reducidos, la formación de los educadores en el ejercicio de sus funciones y el diseño de espacios adecuados. Algunas de estas prácticas fueron señaladas también por exresidentes, entre las que destacan: condiciones organizativas de los centros, estabilidad de los educadores (para generar confianza y poder establecer vínculos referenciales reparadores emocionalmente), cualidades personales del educador, disponibilidad, apoyo, comprensión, afecto y tacto educativo (García-Barriocanal et al., 2007). Entre los conocimientos que se aconseja al educador dominar se encuentran aspectos psicológicos, pedagógicos y antropológicos (Romans, 2000; Vallés, 2009); criterios diagnósticos de psicopatología (Vallés, 2009); las fases del proceso de duelo debido a la separación (Fernández del Valle \& Fuertes, 2007); así como estrategias para el autocuidado (Cruz, 2009; Romans, 2000).

\section{Pedagogía y educación social}

Ante la relativa novedad de las disciplinas existe dificultad para diferenciarlas. Pantoja (1998) considera a la pedagogía social como una disciplina que fundamenta a la profesión de la educación social. Sáez (2007), en su obra: Pedagogía Social y Educación Social, coincide 
en pensar que la pedagogía social es un campo científico y de conocimiento que pretende dar razón de ser a una profesión. La pedagogía social tendrá entonces una triple dimensión: de ciencia, disciplina y materia de enseñanza. La educación social es el "campo de prácticas educativas en el marco social" (p.5). Ortega (2005) coincide con la mayoría de los estudiosos del tema al considerar a la educación social como "el fenómeno, la realidad, la praxis" (p.114) y a la pedagogía social como "la reflexión científica, la disciplina científica, que considera, conceptúa e investiga esa educación social" (p.114).

Dentro de las recomendaciones realizadas a los educadores como resultado del estudio de exresidentes, se mencionan la demanda de implicación emocional por parte del educador y la exigencia de "conocimientos teóricos y prácticos de contenido psicológico, educativo y de intervención social" (García-Barriocanal et al., 2007, p. 165).A este respecto, algunos países de Europa y de América Latina han Ilevado a cabo esfuerzos para delimitar las características de un profesional capaz y bien entrenado para atender de manera directa a los grupos vulnerables, incluidos los niños, adolescentes y adultos (AIEJI, 2007), desde una perspectiva de educación para la vida, denominado educador social. Asociaciones como la Internacional de Educadores Sociales (AIEJI) fundada en Alemania hace más de medio siglo y la Asociación Estatal de Educación Social (ASEDES) en Barcelona, entre otras, han trabajado arduamente en la creación de un marco teórico, metodológico y jurídico para una educación concebida como "la teoría de cómo las condiciones psicológicas, sociales, materiales y diferentes orientaciones de valores promueven o dificultan el desarrollo, el crecimiento, la calidad de vida y el bienestar del individuo o del grupo" (AIEJI, 2005, p.5). Esta teoría es denominada educación social, la que, de acuerdo con Camors (2005), es una "perspectiva particular de la educación general" (p.14).

A pesar de ser un tema relativamente nuevo y no del todo conocido o reconocido, se han llevado a cabo acciones con el fin de defenderlo y desarrollarlo en un espacio profesional "ante marcos institucionales que ignoran y desconocen su lugar" (Camors, 2005, p.18). Cataluña, Andalucía, Castilla-León y Asturias se han dedicado al "educador especializado" y al "educador de calle" (Valero, Cavalle, \& Gimeno, 1989). En el 2005, la Agencia Nacional de Evaluación de la Calidad y Acreditación (ANECA) publicó en Madrid, el Libro Blanco con la finalidad de adaptarlo al espacio europeo.

Poco a poco y de manera relativamente rápida, las recomendaciones internacionales y las acciones para adaptarlas que han realizado otros países han llegado a América. En México, sin embargo, los educadores de albergues infantiles no cuentan con un reconocimiento profesional (Núñez, 2008). Algunos no poseen títulos de educación superior y, en el mejor de los casos, provienen de perfiles académicos variados, agrupándolos por denominaciones que tienen que ver más con su posición dentro del organigrama institucional que con las funciones que desempeñan.

A nivel nacional, el Comité de Normalización de Competencia Laboral (CONOCER) en un intento por promover la profesionalización del recurso humano del sector de asistencia social, lanzó en 2007 la "Norma Técnica de Competencia Laboral (NTCL) en la atención a niños y niñas en centros de atención infantil y la NTCL en el cuidado de niñas, niños y adolescentes en casas hogar, albergues e internados" (CONOCER, 2007). A pesar de ser excelentes estos esfuerzos iniciales que consisten en dotar al personal de algún tipo de capacitación, se pueden considerar aún escuetos en cuanto al contenido. En el estado de Sonora los esfuerzos han sido aun menores. Núñez (2008) llevó a cabo un estudio sobre la niñez vulnerable y cautiva, que abordó el tema de la pertinencia de la profesionalización del personal en internados para menores de escasos recursos, distinguiéndolo como un "facilitador de los procesos de formación, ajeno a los roles tradicionales del docente" (p.6).

\section{Competencias y funciones}

El educador social debe ser competente, poseer aptitudes y técnicas (Ayerbe, 2000) y para su ejercicio requiere de una formación tanto 
en "habilidades como en supuestos teóricos, históricos y culturales" (Sáez, 2005, p.137). Mas este cúmulo de conocimientos se debe concretar con el fin de hacerlo asequible, evaluable y medible. La plataforma para las educadoras y educadores sociales en Europa, define el término competencia como el potencial de acción que el educador social tiene respecto a una "tarea, situación o contexto del trabajo socioeducativo que abarca el conocimiento y las aptitudes intelectuales, manuales y sociales, así como las actitudes y la motivación" (AIEJI, 2005, p.8). Esta definición incluye las características señaladas por ANECA en cuanto que las competencias enfatizan el carácter teórico práctico porque comportan "todo un conjunto de conocimientos, procedimientos, actitudes y capacidades que son personales y se complementan entre sí" (ANECA, 2005, p.54).

ANECA concluyó que las competencias con las que un educador social debe contar son 20 transversales (generales) divididas en 7 instrumentales, 5 interpersonales y 8 sistémicas; y 26 competencias específicas. Estas 46 competencias son consideradas más o menos significativas de acuerdo con el perfil específico que el educador social desarrolle, puesto que los ámbitos de acción de la educación social son diversos. Debido a que en varios países no se cuenta con estudios normalizados en educación social, no a todos los educadores sociales se les puede evaluar en cuanto a competencias específicas se refiere. Sin embargo, todos los profesionales que trabajan en intervención social con menores sí deben poseer las competencias generales para poder desarrollar su trabajo. Dos son las competencias a las que la mayoría de autores consideran imprescindibles: habilidades interpersonales (Barudy, 2005; García-Barriocanalet al., 2007; Melendro, 2007; Mondragón \& Trigueros, 2004) y el compromiso con la identidad (Barudy \& Dantagnan, 2005; Cruz, 2009; Oriana, Montalvo, Min Min, \& Oza, 2006).

Por habilidad interpersonal se entiende a aquella capacidad de expresar los sentimientos, de "relacionarse positivamente con otras personas a través de una escucha empática y de la expresión clara y asertiva de lo que se piensa y/o siente por medios verbales y no verbales" (ANECA, 2005, p. 145). Estas habilidades son las que lograrán que el educador se vincule de manera afectiva particularmente con el niño o adolescente, vinculación que dará paso al logro de la función educativa, pues los niños siempre aprenden para alguien (Barudy, 2005).

El compromiso con la propia identidad es la capacidad para reconocerse y valorarse como profesional que ejerce un servicio a la comunidad y se preocupa por su actualización" (Ibídem, p. 146). Ésta permitirá que el educador social busque su propia formación de manera permanente y realice su trabajo con un sentido de mayor logro personal.

\section{Contenidos de la formación}

Distintas entidades y especialistas concuerdan en que la formación, para este colectivo, es una necesidad (Cruz, 2009; Fernández-Millán, Hamido, \& Fernández-Nava, 2008); si bien no se puede afirmar que exista una relación entre experiencia y competencia profesional, pues ni la experiencia ni la formación por sí mismas garantizan que el educador realice bien su trabajo (Santibañez, 1999). Se debe conocer, no obstante, la formación de los actuales educadores sociales, sus conocimientos, competencias y funciones (Cruz, 2009) puesto que el trabajo desempeñado sobre un marco teórico dotará a los educadores "de unas formas de hacer ágiles, aparentemente intuitivas, que utilizan las estrategias y herramientas educativas disponibles para ir adecuándose en cada momento a las necesidades precisas del joven" (Melendro, 2007, p.156).

En este sentido, la presente investigación reporta un estudio sobre las características de formación con que cuentan los educadores infantiles, entendiendo por características de formación: a) su nivel de estudios; b) antigüedad y c) edad. Además, la relación entre las características personales y de formación de los mismos con el ambiente institucional de los albergues que operan en la ciudad de Hermosillo. 


\section{Método}

\section{Participantes}

Se encuestó a los 117 educadores de 7 instituciones de asistencia social privada y 3 instituciones de asistencia social pública, dedicadas al albergue de menores maltratados o en situación de riesgo o pobreza de la ciudad de Hermosillo, Sonora, México. En el estudio se consideraron a todos los educadores sociales que cada uno de los albergues tenía en el momento del levantamiento de datos. El 23\% fueron varones y el $77 \%$ mujeres. La media de edad para el total de la población de educadores es de 38 años. Sin embargo, algunos albergues manejan un personal tan escaso y de edad tan avanzada que la media supera los 50 años de edad. La antigüedad del personal tiende a estar relacionada con la edad. El personal del Instituto Kino y Hogares Providencia, que tienen una media de edad arriba de 50, también poseen personal entre 20 y 50 años trabajando dentro de la misma institución. El 42\% (de un total de 50) de los educadores tienen licenciaturas relacionadas con el trabajo que se realiza en los albergues infantiles; sólo 38 de ellos (32.4\%) están relacionados directamente con el cuidado de niños. El otro 9.6\% lo conforman licenciaturas como derecho, enfermería y trabajo social.

\section{Instrumentos}

Para el estudio de la formación de los educadores se elaboró una escala de evaluación basada en los indicadores establecidos por el Libro Blanco (ANECA, 2005) con modificaciones. Se escogieron de ellas dos competencias transversales y una específica.

Escala ELES. La escala está conformada por 20 reactivos distribuidos en dos factores: competencias de los educadores y ambiente institucional, presentados en escala de tipo Likert. Para las dimensiones que evalúan las competencias de los educadores vade 0 a 4 donde 0 equivale a totalmente en desacuerdo y 4, totalmente de acuerdo; para las mediciones de ambiente institucional la escala va de 0 a 6 , donde 0 equivale a inadecuado y 6 excelente.
El factor de competencias del educador, compuesto por las dimensiones de habilidades, funciones educativas, actitudes y compromiso con la propia identidad y el factor ambiente institucional está conformado por las variables satisfacción de las necesidades del personal y lenguaje y razonamiento. La escala muestra una consistencia interna de alfa igual a .74.

Las dimensiones a evaluar fueron:

Actitudes. La escala está formada por cuatro ítems basados en el Cuestionario para Profesores (Doménech, Esbrí, González, \& Miret, 2004) con una confiabilidad de consistencia interna de .66. El cuestionario está diseñado para medir las actitudes de los profesores hacia los niños con necesidades educativas especiales derivadas de discapacidad, por lo que se realizaron algunos cambios en los reactivos.

Funciones. Se tomaron tres de las funciones del educador social propuestas por ANECA (2005) y Vallés (2009), tal como fueron plasmadas en el inventario utilizado por Escarbajal (2009) en su Modelo de Cuestionario, para medir los objetivos de actuación profesional. La teoría habla de que las funciones de los educadores deben definirse; en este caso se miden las educativas, reportando un alfa de 86 .

Compromiso con la propia identidad. Se identifica con el sentido de pertenencia e identificación con el trabajo realizado. Consta de cuatro reactivos tomados de la Escala de Clima Organizacional (Franco, 2009) con modificaciones; el alfa fue de .60. La escala utilizada es tipo Likert de 0 a 4 , donde 0 equivale a totalmente en desacuerdo y 4 a totalmente de acuerdo, al igual que en las dimensiones anteriores.

Habilidades. Son consideradas como la capacidad de entender a los demás y poder expresar sentimientos y emociones. Se tomaron cuatro items de la escala de competencias de comunicación interpersonal (Rubin \& Martin, 1994) que mostraron un coeficiente alfa de .77. Los reactivos están diseñados para medir las habilidades de empatía, asertividad y 


\section{Resultados}

expresividad con la misma escala Likert. Tres de ellos con dirección positiva y uno, negativa, mismo que fue posteriormente invertido.

Ambiente. Se agregaron cinco ítems de la Early Chilhood Environment Rating Scale - Revised (ECERS-R) que presenta un alfa de entre .86 a .90. Se eligieron las dimensiones de lenguaje y razonamiento, que se refiere a la utilización de materiales para el desarrollo del lenguaje de los niños y la promoción del lenguaje para el desarrollo de habilidades de razonamiento de la que se excluyeron la mitad de los ítems, dando un total de dos, y cuatro ítems de la dimensión satisfacción de necesidades del adulto, a la que se le realizaron pequeñas modificaciones con el fin de medir el grado de satisfacción que el educador tiene hacia su institución.

\section{Procedimiento}

En cada una de las instituciones se concertó una entrevista con el director o directora del albergue con el fin de solicitar permiso para desarrollar el estudio y explicar los objetivos del mismo. Todos fueron entrevistados por psicólogas entrenadas en la técnica de entrevista y se les explicaron los objetivos y propósito general de la investigación, asegurándoles la confidencialidad de la información, así como la posibilidad de dejar de contestar en el momento que ellos dispusieran. La entrevista tomó alrededor de 25 minutos.

\section{Análisis de datos}

Por medio del paquete estadístico SPSS, se realizaron análisis univariados que incluían la obtención de medias y desviaciones estándar para las variables continuas, frecuencias para las variables categóricas y alfas de Cronbach para determinar su confiabilidad (consistencia interna). Con el fin de agrupar las variables, se formaron índices con la suma de cada uno de los reactivos de las escalas incluidas en el estudio. También se llevó a cabo un análisis de correlación de Pearson para estimar el grado de asociación entre las variables, considerando una $\mathrm{p} \leq .05$ para determinar su significancia.
Los resultados de la aplicación de la Evaluación de la labor del educador social (ELES) mostraron un coeficiente de confiabilidad de .80 (tabla 1). Aun cuando las alfas de tres dimensiones reportaron alfas menores a .60, se decidió conservarlas.

En la tabla 2 se pueden ver los coeficientes de correlación de Pearson hallados entre las escalas, en donde se encontraron correlaciones positivas en su mayoría. Lenguaje y razonamiento se correlacionó con funciones educativas (.26) y compromiso con la propia identidad (26), satisfacción de necesidades con compromiso con la propia identidad (.22) y lenguaje y razonamiento (.39), último grado de estudios con habilidades interpersonales (26). La edad se correlacionó con la antigüedad (.48), último grado de estudios (-.19), satisfacción de necesidades (.25) y lenguaje y razonamiento (.19).

\section{Discusión}

La profesión del educador social refleja su propia historia (Vallés, 2009). Lo hizo en Alemania, en España y lo hace en América Latina. Los resultados de este estudio son prueba de ello puesto que la especialización del educador en esta rama de la educación no emerge de las universidades sino de la propia práctica de cientos de personas dispuestas a aprender a educar y cuidar a los niños y adolescentes en problemas. Es por esto que resulta importante hacer notar que no sólo la formación profesional garantiza la adecuada labor socioeducativa (Santibañez, 1999), sino que es necesaria una educación especializada (Barjau, 2001, Fernández del Valle \& Fuertes, 2007; Romans, 2000). Cabe mencionar que la mayoría de los educadores con menor formación profesional tienen más tiempo de antigüedad. Aquéllos que llevan de 15 a 50 años en este trabajo son los educadores con estudios de primaria y secundaria solamente. Quienes tienen de 10 a 15 años son educadores con estudios de preparatoria y licenciaturas no relacionadas con la labor de los albergues. Aquéllos más profesionalizados han sido más contratados. 


\section{Tabla 1}

Análisis de confiabilidad de las escalas de ELES y medias y desviaciones estándar de cada uno de los reactivos

\begin{tabular}{|c|c|c|c|c|}
\hline Variable & $\mathrm{N}$ & Media & D.E. & Alfa \\
\hline ACTITUDES & & & & .39 \\
\hline Mi trato cordial con los niños y niñas estimula su desarrollo & 116 & 3.83 & .40 & \\
\hline Los niños y niñas del albergue no tienen capacidad para aprender & 117 & 3.67 & .79 & \\
\hline $\begin{array}{l}\text { Los niños no pueden enfrentarse a los desafíos que les plantea el sistema } \\
\text { social }\end{array}$ & 115 & 2.63 & 1.49 & \\
\hline La integración social favorece a los niños y niñas del albergue & 115 & 3.70 & .80 & \\
\hline FUNCIONES EDUCATIVAS & & & & .62 \\
\hline Educativa & 117 & 3.50 & 1.03 & \\
\hline Informativa, de asesoramiento, orientadora & 115 & 3.64 & .77 & \\
\hline De animación y dinamización de grupos & 115 & 3.74 & .72 & \\
\hline COMPROMISO CON LA PROPIA IDENTIDAD & & & & .46 \\
\hline Siento que mi trabajo es bien recibido por mis compañeros y compañeras & 117 & 3.49 & .83 & \\
\hline Les platico a mis familiares y amigos cuánto me gusta mi trabajo & 115 & 3.65 & .81 & \\
\hline Siento que el pertenecer a esta área de trabajo me da algo más que dinero & 116 & 3.90 & .30 & \\
\hline $\begin{array}{l}\text { Considero que mis compañeros/as y yo, nos sentimos identificados/as con } \\
\text { nuestra área de trabajo }\end{array}$ & 117 & 3.68 & .67 & \\
\hline HABILIDADES INTERPERSONALES & & & & .57 \\
\hline No entiendo realmente lo que los otros sienten & 116 & 3.50 & .91 & \\
\hline Tengo problemas para defenderme a mí mismo/a & 116 & 3.28 & 1.30 & \\
\hline Se me dificulta encontrar las palabras correctas para expresarme & 117 & 2.98 & 1.46 & \\
\hline Me expreso bien verbalmente & 117 & 3.56 & .83 & \\
\hline LENGUAJE Y RAZONAMIENTO & & & & .91 \\
\hline La estimulación a los niños para comunicarse es & 116 & 4.47 & 1.34 & \\
\hline La utilización del lenguaje para desarrollar destrezas de pensamiento es & 114 & 4.14 & 1.61 & \\
\hline SATISFACCIÓN PERSONAL & & & & .84 \\
\hline La disposición para atender necesidades individuales del personal & 116 & 3.99 & 1.71 & \\
\hline El apoyo para necesidades profesionales del personal & 115 & 3.68 & 1.89 & \\
\hline $\begin{array}{l}\text { Las oportunidades de crecimiento y carrera profesional dentro de la } \\
\text { Institución }\end{array}$ & 115 & 3.43 & 1.92 & \\
\hline
\end{tabular}


Tabla 2

Correlación entre competencias del educador, características de formación y ambiente institucional

\begin{tabular}{|c|c|c|c|c|c|}
\hline \multirow[b]{2}{*}{ Competencias } & \multicolumn{2}{|c|}{ Ambiente Institucional } & \multicolumn{3}{|c|}{ Características Personales } \\
\hline & $\begin{array}{l}\text { Lenguaje y } \\
\text { Razonamiento }\end{array}$ & $\begin{array}{l}\text { Satisfacción de } \\
\text { las Necesidades }\end{array}$ & Edad & $\begin{array}{l}\text { Último Grado de } \\
\text { Estudios }\end{array}$ & Antigüedad \\
\hline Actitudes & .103 & .105 & -.046 & .080 & .065 \\
\hline $\begin{array}{l}\text { Funciones } \\
\text { educativas }\end{array}$ & $.262^{\star *}$ & .108 & 0.35 & .079 & -.065 \\
\hline $\begin{array}{l}\text { Habilidades } \\
\text { interpersonales }\end{array}$ & .088 & -.068 & -.051 & $.263^{\star *}$ & -.002 \\
\hline $\begin{array}{l}\text { Compromiso con la } \\
\text { propia identidad }\end{array}$ & $.265^{\star \star}$ & $.220^{*}$ & .145 & .048 & .102 \\
\hline \multicolumn{6}{|l|}{$\begin{array}{l}\text { Ambiente } \\
\text { institucional }\end{array}$} \\
\hline $\begin{array}{l}\text { Lenguaje y } \\
\text { razonamiento }\end{array}$ & & $.395^{\star *}$ & .196 & -.033 & .167 \\
\hline $\begin{array}{l}\text { Satisfacción de las } \\
\text { necesidades }\end{array}$ & .395 & & .252 & .087 & .151 \\
\hline edad & $.196^{\star}$ & $.252^{\star *}$ & & $-.191^{\star}$ & $.483^{\star *}$ \\
\hline Grado de estudios & -.033 & .087 & -.191 & & -.077 \\
\hline Antigüedad & .167 & .151 & .483 & -.077 & \\
\hline
\end{tabular}

Con relación a lo anterior, resaltan dos aspectos: primero, que con el transcurso del tiempo se fue tendiendo hacia la contratación de un educador más profesionalizado de acuerdo con las recomendaciones internacionales (Gobierno de Brasil, 2007; UNICEF, 2002) y nacionales (Cámara de Diputados, 2009; Castro, 1996); segundo, se podría pensar que, puesto que los educadores con menor formación profesional tienen mayor antigüedad, han tenido también más experiencias de historias de éxito entre los menores que han atendido, lo que les permitiría exhibir una actitud más positiva hacia ellos. Sin embargo, esta hipótesis queda abierta para futuras investigaciones.

Las correlaciones con las dimensiones de ambiente institucional encontradas en este estudio resultan también interesantes. Originalmente, la variable satisfacción del personal se pensó importante puesto que algunos especialistas consideran que las condiciones laborales inadecuadas pueden tener una influencia negativa en el desarrollo de la práctica educativa (Cruz, 2009). Esta situación puede tornar el ambiente en poco acogedor y afectar a los menores vulnerables (Melendro, 2007). Además, los educadores requieren sentirse respetados en sus centros de trabajo y que sus necesidades, dificultades y logros sean reconocidos (Barudy \& Dantagnan, 2005). Lo que finalmente evitará el desgaste profesional y los efectos indirectos que éste pudiera tener en los menores. Sin embargo, existe una correlación entre ambiente institucional y compromiso con la identidad lo que parece indicar que contar con un buen ambiente institucional, ayuda a que los educadores desarrollen un mayor compromiso con el trabajo que realizan. Esto es importante para los niños que se encuentran en los albergues, ya que les posibilita recibir relaciones positivas por parte de los educadores, para generar esquemas sanos de comportamiento y relaciones interpersonales efectivas para una convivencia adaptativa y prosocial (Mondragón \& Trigueros, 2004, p. 196).

Por otro lado, lenguaje y razonamiento se correlaciona con funciones educativas, lo que denota que si se definen las funciones del educador como educativas estos van a invertir en más actividades de promoción del lenguaje y de razonamiento. Los educadores en los alberques realizan todo tipo de actividades, muchas veces administrativas que los desvían de las educativas. El definir sus actividades como 
educativas va a motivar en ellos el promover tareas de lenguaje y razonamiento en los niños. Los niveles de estudio están relacionados con las habilidades interpersonales, lo que indicaría que a mayor educación recibida por ellos, mayores sus habilidades interpersonales. Estas habilidades son esenciales en los educadores, ya que les ayuda a los niños a sentir la proximidad y afecto que los impulsa a establecer relaciones interpersonales dinámicas y duraderas (Melendro, 2007). La estancia en los albergues puede estimular o desalentar el desarrollo social, emocional y psicológico del niño; un ambiente positivo lo estimulará, en cambio el negativo producirá conducta desadaptada (Wachs, 2000), bajo rendimiento escolar (Reyes Mendoza \& Solis Lugo, 1999) retrasos en el desarrollo, depresión, ansiedad y dificultades en el aprendizaje (Bassuk \& Rubin, 1987), además de problemas emocionales y cognitivos (Rescorla et al., 1991). En general, los niños de albergue, manifestaban más problemas de internalización y externalización (Bassuk et al., 1997). Por esto, es importante que los educadores que trabajan en los albergues estén preparador para poder proporcionar el ambiente positivo que se requiere para promover el desarrollo de los niños.

A pesar de que este estudio no ahondó en todas las competencias sugeridas por ANECA, sí se sugiere su estudio y profundización como contenido de un curso de formación para los educadores. Se sustenta la idea de que estos educadores podrían contar con más herramientas para el desarrollo eficaz de su labor al ser formados en una teoría educativa pertinente (AIEJI, 2005; ANECA, 2005; Sáez, 2005), tal como es la de Pedagogía Social/ Educación Social, posiblemente a través de la creación de una licenciatura o grado académico de pos-grado.

En aras de mejorar la labor educativa de los albergues, los resultados de este estudio permiten establecer la necesidad de continuar con investigaciones que busquen dar luz a ciertas interrogantes. Una de ellas es analizar por qué los educadores con mayor profesionalización tienen actitudes menos positivas hacia los menores y menos habilidades interpersonales. Sería interesante ahondar también en la capacidad de vinculación que tienen los educadores y la experiencia que de ellos guardan aquellos niños y adolescentes, ahora adultos. Como colofón a esta investigación, se podría analizar de igual manera si los cursos impartidos a directores y educadores como fruto de este estudio tuvieron algún impacto en la práctica educativa de los albergues.

\section{PSICUMEX}




\section{Referencias}

Achenbach, T. (1992). Manual for the Child Behavior Checklist/2-3 and 1992 Profile. Burlington, VT: University of Vermont Department of Psychiatry.

Agencia Nacional de Evaluación de la Calidad y Acreditación (ANECA). (2005). Libro Blanco. Título de grado en pedagogía y educación social. Volumen 1. España: ANECA.

Arruabarrena, M. (2001). Evaluación y tratamiento familiar. En J. De Paul y M. Arruabarrena (Eds.), Manual de protección infantil (pp. 297340). Barcelona: Massons.

Asociación Internacional de Educadores Sociales. (AIEJI). (2005). Plataforma para laseducadoras $y$ educadores sociales en Europa. España: AIEJI. Recuperado de http://www.ceesc.cat/ index2.php?option $=$ com_docman\&task $=$ doc_ view\&gid $=75 \&$ Itemid $=403$

Asociación Internacional de Educadores Sociales. (AIEJI). (2007). Estatutos de AIEJI. Barcelona: AIEJI. Recuperado de http://www. aieji.net/attached/bylaws/By-Laws2007SFinal. pdf

Ayerbe, P. (2000). La figura del educador social y otros profesionales de la educación. En P. Amorós y P. Ayerbe (Eds.), Intervención educativa en inadaptación social (pp. 55-81). Madrid: Síntesis.

Barjau, C. (2001). Acogimiento familiar, un medio de protección infantil. En J. De Paul y M. Arruabarrena (Eds.), Manual de protección infantil (pp. 375-408). Barcelona: Massons.

Barudy, J. (2005). La ecología social de los buenos tratos infantiles. En J. Barudy y M. Dantagnan (Eds.), Los buenos tratos a la infancia (pp. 43-52). Barcelona: Gedisa.

Barudy, J., \& Dantagnan, M. (Eds.). (2005). Un modelo terapéutico basado en el buen trato y el apoyo a la resilencia. En Los buenos tratos a la infancia (pp. 213-244). Barcelona: Gedisa.
Bassuk, E., \& Rubin L. (1987). Homeless children: A Neglected Population. American Journal of Orthopsychiatry, 57(2), 279-286. doi: 10.1111/ j.1939-0025.1987.tb03538_x.

Bassuk, E. L., \& Rosenberg, L. (1990). Psychosocial Characteristics of Homeless Children and Children With Homes. Pediatrics, 85(3), $257-261$.

Bassuk, E. L., Weinreb, L. F., Dawson, R., Perloff, J.N., \& Buckner, J.C. (1997). Determinants of Behavior in Homeless and Low-income Housed Preschool Children. Pediatrics, 100(1), 92 -100. doi: 10.1542/peds.100.1.92.

Bradley, R. H. (2002). Environment and Parenting. En M. H. Bornstein, (Ed.), Handbook of Parenting, Vol. 3.Social Ecology of Parenting (pp. 281-314). Mahwah, NJ.: Lawrence Erlbaum Associates, Publishers.

Bravo, A., \& Fernández Del Valle, J. (2009). Crisis y revisión del acogimiento residencial. Su papel en la protección infantil. Papeles del Psicólogo, 30(1), 42-52.

Cámara de Diputados (2009). Iniciativa de Diputados del PRI para operación de Casas Hogar. Carpeta Informativa. Coordinación General de Comunicación Social . LX Legislatura. Recuperado de http://www. cddhcu.gob.mx.

Camors, J. (2005). Educación social: una perspectiva desde y para América Latina. En XVI Congreso Mundial de Educación Social. Montevideo, Uruguay. Recuperado de http://74.125.155.132/ search?q=cache:EABjOEG9XXoJ:www. projoven.gub.uy/pag-otra-doc-relev/ Documentos/Congreso\%2520educadores/1_ Jorge_ucaci\%C3\%B3n+social:+una+perspe ctiva+desde+y+para+Am\%C3\%A9rica+Lati $n a+\% 2 B+C a m o r s \& c d=1 \& h l=e s \& c t=c \mid n k \& g l=$ $\mathrm{mx \&}$ client=firefox-a

Castro, P. K. (noviembre, 1996). Un problema que a todos incumbe. En Congreso Nacional sobre Maltrato al Menor. Memorias. 26, 27 y 28 de noviembre 1995 (26-28). México: Sistema Nacional para el Desarrollo Integral de la Familia. 
Cervantes, E. (1997). El papel del psicólogo en la planeación y organización de centros asistenciales para menores maltratados (Tesis de licenciatura inédita). Universidad de Sonora, Hermosillo, Sonora, México.

Chávez, F. (1986). El Trabajador social y las instituciones de asistencia social para menores maltratados y/o abandonados. (Tesis de licenciatura inédita). Universidad de Sonora, Hermosillo, Sonora, México.

Comunidad Valenciana. (1991). Estatuto básico de centros de atención a la infancia y juventud en la comunidad valenciana. Diari Oficial de la Comunitat Valenciana (Orden de 14 de mayo de 1991), Consellería de Trabajo y Seguridad Social. Recuperado de http:// www.docv.gva.es/portal/ficha_disposicion_ pc.jsp?sig=1369/1991\&L=1

CONOCER. (2007). Norma Técnica de Competencia Laboral. México. Recuperado de http://www.conocer.gob.mx/Desarrollo/ Buscadores_Avanzados/pdf/NUASS006.01. pdf

Contreras, J. (febrero, 2009). Pide Senado investigar a Casitas del Sur y a la Iglesia Cristiana Restaurada. La Crónica de Hoy. México. Recuperado de http://www.cronica. com.mx/nota.php?id_nota $=414133$

Cruz, L. (2009). Infancias y educación social: prácticas socioeducativas en contextos residenciales de protección en Galicia. (Tesis Doctoral). Universidad de Santiago de Compostela, España.

Doménech, V., Esbrí, J., González, H., \& Miret, L. (2004). Actitudes del profesorado hacia el alumnado con necesidades educativas especiales derivadas de discapacidad. Novenes Jornades de Foment de la Investigació, 9, 1 - 13. Recuperado de http:// www.uji.es/publ/edicions/jfig/

Dupper, D. R., \& Halter, A. P. (1994). Barriers in Educating Children from Homeless Shelters: Perspectives of School and Shelter Staff. Children Schools, 16(1), 39-45. doi: 10.1093/ cs/16.1.39.
Escarbajal, A. (2009). Estudio de las respuestas socioeducativas ofrecidas a los inmigrantes en la región de Murcia y propuesta formativa para trabajadores sociales y educadores sociales. (Tesis doctoral).Universidad de Murcia, Murcia, España.

Fernández del Valle, J. (1992). Evaluación de programas residenciales y servicios sociales para la infancia: situación actual y aportaciones de los enfoques ecopsicológicos. Psicothema, 4(1), 531-542.

Fernández del Valle, J., \& Fuertes, J. (2007). El acogimiento residencial en la protección a la infancia. España: Pirámide.

Fernández-Millán, S., Hamido, A., \& FernándezNava, M. (2008). El educador social de menores. Cuaderno de aprendizaje. Madrid: Pirámide.

Franco, J. (2009). Validación de una escala de clima organizacional en el sector de servicios en el Noroeste de México (Tesis de licenciatura inédita). Universidad de Sonora, Hermosillo, Sonora, México.

Fuertes, J., \& Fernández del Valle, J. (2001). Acogimiento residencial. En J De Paul y M. Arruabarrena (Eds.), Manual de protección infantil (pp. 409-470). Barcelona: Massons.

García-Barriocanal, C.; De la Herrán, A., \& Imaña, A. (2007). El acogimiento residencial como medida de protección. Madrid: Defensor del menor en la Comunidad de Madrid.

Gobierno de Brasil. (2007). Anteproyecto de Naciones Unidas para el uso apropiado y condiciones del cuidado alternativo de niños/ as. Aprobada en la Asamblea General de Naciones Unidas el 20 de noviembre de 2009. Recuperado de http://www.crin.org/docs/ DRAFT_UN_Guidelines.pdf

Gobierno de México. (2002). Estado de México: Comité Editorial de la Administración Pública Estatal.

Ley de Salud para el Estado de Sonora. (1992). Gobierno del Estado de Sonora. 
Melendro, M. (Dir.). (2007). Estrategias educativas con adolescentes y jóvenes en dificultad social. El tránsito a la vida adulta en una sociedad sostenible. España: UNED.

Melendro, M. (Dir.). (2010). El tránsito a la vida adulta de los jóvenes en dificultad social. España: UNED.

Mondragón, J., \& Trigueros, I. (2004). Intervención con menores. Acción socioeducativa. Madrid: Narcea.

Nesmith, A. (2006). Predictors of Running way from Family Foster Care. ChildWelfare, 85(3), 585-609.

Núñez, V. M. M. (2008). Intervención Interdisciplinaria para una Niñez Vulnerable y Cautiva (Tesis de Maestría inédita). Universidad de Sonora, Hermosillo, Sonora, México.

Oriana, L., Montalvo, D., Min Min, L., \& Oza, V. (2006). A promising parenting intervention in foster care. Journal of Consulting and Clinical Psychology, 74(1), 32-41.

Ortega, J. (2005). Pedagogía social y pedagogía escolar: la educación social en la escuela. Revista de Educación, 336, 111 $-117$.

Pantoja, L. (1998). Nuevos espacios de la educación social. Bilbao: Ediciones Mensajero, S.A.

Papalia, D.,Wendkos, S., \& Duskin, R. (2001). Desarrollo Humano. Bogotá: McGraw-Hill Interamericana.

Redondo, E., Muñoz, R., \& Torres Gómez. (1998). Manual de buenas prácticas para la atención residencial a la infancia y adolescencia. Madrid: FAPMI.

Rescorla, L., Parker, R., \& Stolley, P. (1991). Ability, Achievement, and Adjustment in Homeless Children. American Journal of Orthopsychiatry, 61(2), 210-220. doi: 10.1037/h0079236.
Reyes Mendoza, N. M., \& Solis Lugo, A. T. (1999).Rendimiento académico de niños que viven en un albergue o en hogares propios. Educación y Ciencia, 3(5), 91-98.

Romans, M. (2000). Formación continua de los profesionales en Educación Social. En M. Romans, R. Petrus y J. Trilla, Nuevos ámbitos en Educación Social (pp. 151-277). Barcelona: Paidós.

Rubin, R., \& Martin, M. (1994). Development of a Measure of Interpersonal Communication. Competence. Communication. Research Reports, 11(1), $33-44$.

Sáez, J. (2005). La profesionalización de los educadores sociales: construcción de un modelo teórico para su estudio. Revista de Educación, 336, 129-139.

Sáez, J. (2007). Pedagogía social y educación social. Historia, profesión y competencias. Madrid: Pearson Educación, S.A.

Santibañez, R. (1999). El seguimiento en el practicum de Educación Social. En F. Esteban y R. Calvo de León, El practicum de la formación de Educación Social (pp. 189200). Burgos: Servicio de Publicaciones de la Universidad de Burgos.

Schreiner, G. (2009). Riesgo o abandono: más allá de la semántica. Recuperado de http:// www.conscienciasocial.net/attachments/

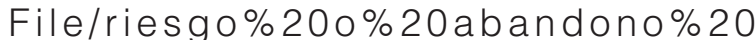
m\%C3\%A1s\%20all\%C3\%A1\%20de\%20 la\%20sem\%C3\%A1ntica\%20_Gabriela\%20 Schreiner.pdf.

UNICEF. (2002). Un mundo apropiado para los niños y las niñas. Sesión Especial a favor de la Infancia. Recuperado de http://www. unicef.org/lac/Un_mundo_apropiado_para_ los_ninos_y_las_ninas.pdf

UNICEF. (2007). La Infancia y los Objetivos de Desarrollo del Milenio. Recuperado de http:// www.unicef.org/spanish/publications/files/ La_infancia_y_los_objetivos_de_desarrollo_ del_milenio.pdf. 
UNICEF. (2009). Progreso para la Infancia. Un balance sobre la protección de la Niñez. Núm 8. Recuperado de http://www.unicef. org/spanish/progressforchildren/files/ Progress_for_Children-No.8_SP_081309. pdf.

Valero, L., Cavalle, J., \& Gimeno, J. (1989). Perspectivas del trabajo para los educadores diplomados en las escuelas de magisterio. Revista Universitaria de Formación del Profesorado, 6, 393-399. Recuperado de http://dialnet.unirioja.es/ servlet/articulo?codigo $=117668$

Vallés, J. (2009). Manual del educador social. Madrid: Pirámide.

Wachs, T. D. (2000). Necessary but not sufficient. Washington, DC: American Psychological Association. 